\title{
An integral heat flux sensor with high spatial and temporal resolutions
}

\author{
Song $\mathrm{Wu} \cdot$ Yong-Hua Shu $\cdot$ Jin-Ping $\mathrm{Li} \cdot$ \\ Hong-Ru Yu
}

Received: 4 September 2013/Accepted: 18 February 2014/Published online: 5 June 2014

(C) Science China Press and Springer-Verlag Berlin Heidelberg 2014

\begin{abstract}
To measure heat flux on the sharp leading edge and other surface with very small curvature radius in the wind tunnel, Hong-Ru Yu proposed a novel design of integral type heat flux sensor. Compared with the traditional heat flux sensor, the integral type heat flux sensor improved spatial resolution up to an order of magnitude, and the size of the sensing elements has been minimized to $0.1 \mathrm{~mm}$ successfully, which satisfied the heat flux measurement requirements at $R 0.5 \mathrm{~mm}$ pointed cone stagnation point. A constantan film was used as the contact layer of the integral heat flux sensor, which was prepared by electron beam coevaporation method. The influence of sensor structure on the accuracy of measurement was analyzed using numerical calculation. The measurement results in shock wave wind tunnel indicated that the response frequency was about $100 \mathrm{kHz}$ and the repeatability error was less than $10 \%$.
\end{abstract}

Keywords Shock tunnel - Heat flux sensor ·

Constantan film · Electron beam co-evaporation

\section{Introduction}

Thermal protection is one of the key issues for the design of hypersonic aircraft, and it is important to know the heat generated on the surface of aircraft by aerodynamic heating, and then suitable heat-resistant materials and reasonable thermal structure designs can be considered. Ground testing is one of the main approaches to predict surface heat flux.

S. Wu (ه) · Y.-H. Shu · J.-P. Li · H.-R. Yu

State Key Laboratory of High Temperature Gas Dynamics, Institute of Mechanics, Chinese Academy of Sciences, Beijing 100190, China

e-mail: wusong@imech.ac.cn
Heat measurement techniques on ground can be divided into two categories: one is based on the heat flow sensors, such as resistance thermometers, thermocouples, calorimeter, etc., and the other one is based on optical heat-measuring techniques, such as infrared thermometry, liquid crystal thermometry, phase change coatings, phosphorescence thermometry and temperature-and pressure-sensitive paint, etc. $[1,2]$. The temperature and heat flux distribution on the aircraft model surface can be directly shown using optical heat-measurement techniques, but the accuracy of the optical measurements in shock tunnel tests can be seriously affected by the impurities of the flow field and the vibration of the model and so on, so the heat flux in shock tunnel is mainly measured using heat flux sensors in the world.

Platinum film resistance thermometers and coaxial thermocouples, usually in cylindrical shape, are often used to measure heat flux in shock tunnel. In Europe, the minimum diameter of coaxial nickel and chromium thermocouples developed by Aachen university is about $1.9 \mathrm{~mm}$ [3], and the film resistance thermometer developed by the Calspan laboratory [4] has a diameter of only $1 \mathrm{~mm}$, which is the smallest cylindrical heat flux sensor used in shock wave wind tunnel. In China, most of the diameter of heat flux sensors is about $2 \mathrm{~mm}$, and the smallest diameter of the coaxial thermocouple is about $1.4 \mathrm{~mm}$ [5], and the film resistance thermometers with a diameter of $1 \mathrm{~mm}$ is being studied. Scaled models are often used in shock tunnel tests. Stagnation points, wing tips, bosses, and cracks are often loaded with high heat flux. After being scaled, the size of models become too small and cylindrical heat flux sensor with $1 \mathrm{~mm}$ diameter cannot satisfy the measurement requirements, so higher spatial resolution heat flux sensor is needed. Planar platinum film resistance thermometer with a sensitive element of about $0.1 \mathrm{~mm}$ width and $1.3 \mathrm{~mm}$ length can be used to measure heat flow of $R 1 \mathrm{~mm}$ 
blunt leading edge stagnation point [6]. But all of the above heat flux sensors cannot meet heat flux measurement requirements of ball cone stagnation point with radius of 0.5 to $5 \mathrm{~mm}$, which were actually concerned in engineering applications.

To resolve this problem, Hong-Ru Yu proposed a novel design of an integral heat flux sensor (Fig. 1a), where a conic shape made of metals such as copper-nickel alloy was used as one pole of the thermocouple, and a wire made of another metals such as nickel-chromium alloy was pulled through the apex was used as the other pole of the thermocouple. An insulating layer was placed between the two poles, and a thermal junction of metal film was prepared on the top of the thermocouples using electron beam coevaporation method. Figure $1 \mathrm{~b}$ is a photo of the integral heat flux sensor developed by the Institute of Mechanics, the Chinese Academy of Sciences. The thermocouple is intended to measure the temperature change across the junction. Therefore, dimensions of the sensing elements used in the integral thermocouple are determined by the wire diameter. In the case of nickel chromium wire, nickel chromium wire with successful test, has achieved a diameter as small as $0.1 \mathrm{~mm}$.

\section{Design principles of the integral heat flux sensor}

The integral heat flux sensor is designed on the basis of thermoelectric effect. At a certain temperature, two different conductor materials will vary in the free electron density. At the junction, due to the free electron diffusion, a

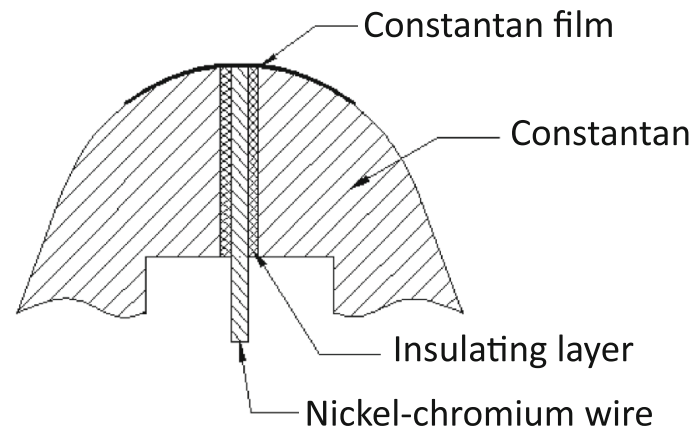

(a)

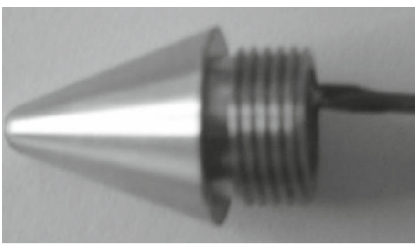

(b)

Fig. 1 Integral conical heat flux sensor. a Schematic diagram; b photo of the real instrument diffusion electromotive force will be generated and the magnitude is dependent on properties of the two materials and the temperature at the contact point.

As shown in Fig. 2, two different materials form a electric loop, and the end with the higher temperature is known as the measurement or the hot end, and that with the lower temperature as the reference or the cold end. Usually, the temperature of the reference end $T_{0}$ remains constant, thus the thermoelectric force is dependent on the temperature of the measurement end $T$ only, that is,

$\left.E_{\mathrm{AB}}\left(T, T_{0}\right)\right|_{T_{0}}=E_{\mathrm{AB}}(T)$.

Since the nickel-chromium wire is very thin, the temperature changes at the measurement end due to aerodynamic heating can be approximated by a one-dimensional semi-infinite heat conduction equation, i.e.,

$\frac{\partial^{2} T}{\partial x^{2}}=\frac{1}{\alpha} \frac{\partial T}{\partial t}$,

where $\alpha=k / \rho c, k$ is the thermal conductivity, $\rho$ is the density, and $c$ is the specific heat.

Suppose the heat flow on the exposing surface of the nickel-chromium wire is $\dot{q}_{s}(t)$, then

$x=0, \quad \dot{q}_{s}(t)=-k \frac{\partial T}{\partial x}$,

$x=\infty, \quad T=T_{0}$.

Based on the above boundary conditions, Eq. (2) can be rewritten as [7]

$q_{\mathrm{s}}(t)=\frac{\sqrt{\rho c k}}{2 \sqrt{\pi}}\left[\frac{2 T(t)}{\sqrt{t}}+\int_{0}^{t} \frac{T(t)-T(\tau)}{(t-\tau)^{3 / 2}} \mathrm{~d} \tau\right]$.

According to Eq. (1) and the measured diffusion force, the temperature history on the nickel-chromium wire

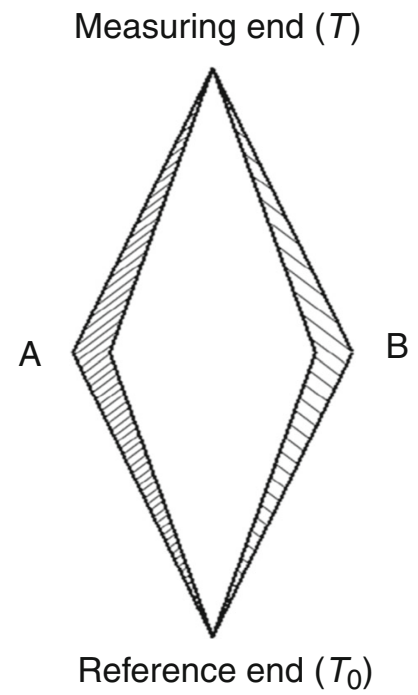

Fig. 2 Schematic diagram for thermometry of a thermocouple 
surface can be obtained with the integral heat flux sensor. Substituting it into Eq. (5), and the heat flux of the cone vertex concerned can be determined.

\section{Constantan thin film prepared by electron beam coevaporation method}

The quality of the thermal junction layer significantly affects performances of the heat flux sensor, where the quality of the thermal junction layer is determined by the film thickness and component distributions. The junction layers prepared using methods such as spot welding, mechanical polishing and chemical plating are difficult to control the film thickness and component distributions. In this paper, thermal junction layer of constantan films was deposited on the surface of the integral heat flux sensor by coevaporation of $\mathrm{Ni}$ and $\mathrm{Cu}$ sources using electron beam physical vapor deposition (EBPVD) method, and the evaporation rates of copper and nickel are controlled preciously.

In the process of EBPVD, evaporation material is heated by high-energy electron beam accelerated in electric field and focused in magnetic field. The material is placed in a crucible cooled by circulating water, and contamination of the crucible material can be avoided, so high-purity films can be prepared using the electron beam evaporation method.

With the support of "the Tenth Five-year Plan" equipment development project of Chinese Academy of Sciences, a multi-source electron beam physical vapor deposition system was developed by the Institute of Mechanics, Chinese Academy of Sciences (IMCAS-MEBPVD). The system is composed of vacuum and gas inlet system, evaporation and control system, substrate movement and heating system, time-of-flight mass spectrometer, and quantitative forecasting software of rare evaporation gas flow field simulation $[8,9]$.

As shown in Fig. 3, the constantan junction thin layer was deposited on the cone heat flux sensor by coevaporation of two materials using two electron guns of the IMCAS-MEBPVD system, and the rate of each evaporation source was monitored and controlled by the corresponding quartz crystal monitors (QCM). Electron gun \# 1 was used to evaporate of $\mathrm{Ni}$ (mass purity $99.999 \%$ ), and the evaporation rate was controlled to $1.8 \AA ̊ \AA / s$ by Inficon quartz crystal monitor (CTC/2). Electron gun \# 2 was used to evaporate of $\mathrm{Cu}$ (mass purity 99.999\%), and the evaporation rate was controlled to $1.8 \AA$ $\AA / \mathrm{s}$ by Inficon quartz crystal monitor (IC/5). Both of the thickness of $\mathrm{Ni}$ and $\mathrm{Cu}$ were set to $6000 \AA$.

The cone heat flux sensors were placed on the substrate holder, and the cone head pointed to the evaporation source. The rotation axis of the substrate holder is connected to a

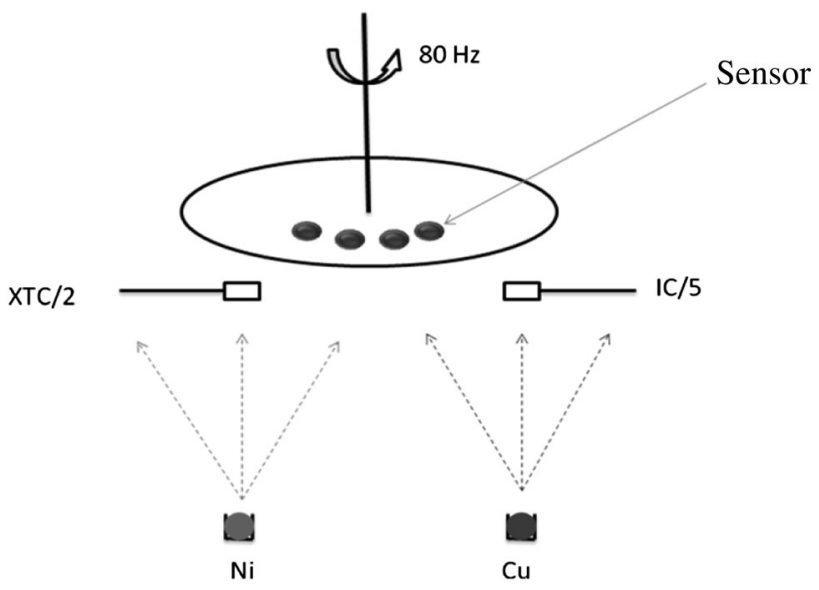

Fig. 3 Schematic diagram of the positions of evaporation sources, quartz crystal probes, and substrate in the vacuum chamber of IMCAS-MEBPVD System

rotating motor installed on the top of the IMCAS-MEBPVD system, and the speed of rotation was about $80 \mathrm{r} / \mathrm{min}$. Before deposition of the constantan junction thin layer, the vacuum chamber was degassed by heating the vacuum chamber, and the pressure is about $5 \times 10^{-4} \mathrm{~Pa}$. After deposition, the molar ratio of $\mathrm{Ni}$ and $\mathrm{Cu}$ of the deposited junction layer was measured using an in-ductively coupled plasma atomic emission spectrometer (ICP-AES), result indicated that the thickness of the film deposited on the surface of the cone heat flux sensor was about $1.2 \mu \mathrm{m}$, and the atomic ratio of $\mathrm{Ni}$ to $\mathrm{Cu}$ was about 45:54.

\section{Response characteristic of the integral heat flux sensor}

The integral heat flux sensor is a structure made of two metals and an insulation layer. Its thermal response characteristic could be obtained through numerical analysis of the heat transfer process.

In the numerical analysis, the governing equation is an unsteady two-dimensional axisymmetric heat conduction equation, discretized and resolved using a finite difference method. In the numerical model, the radius of the head of the integral heat flux sensor is $1 \mathrm{~mm}$, the thickness of the surface constantan film is $2 \mu \mathrm{m}$, and the thickness of the insulating layer between the constantan and nickel-chromium is $20 \mu \mathrm{m}$. The density, specific heat and thermal conductivity of the constantan, nickel-chromium and the insulating layer (epoxy resin) are shown in Table 1. The input heat flux on the sensor surface is set to be $q_{0}=1.0 \mathrm{MW} / \mathrm{m}^{2}$.

Figure 4 shows the calculated temperature curve of the thermal junction layer of the thin constantan. It is slightly higher than the corresponding theoretical value obtained 
Table 1 Material properties of the integral heat flux sensor

\begin{tabular}{llll}
\hline Material property & Constantan & $\begin{array}{l}\text { Nickel- } \\
\text { Chromium }\end{array}$ & $\begin{array}{l}\text { Epoxy } \\
\text { resin }\end{array}$ \\
\hline Density $\left(\mathrm{kg} / \mathrm{m}^{3}\right)$ & 8920 & 8730 & 1060 \\
Specific heat $(\mathrm{J} /(\mathrm{kg} \mathrm{K}))$ & 393.1 & 447.5 & 1960 \\
Heat conductivity $\left(\mathrm{J} /\left(\mathrm{s} \mathrm{m}^{2} \mathrm{~K}\right)\right)$ & 21.17 & 19.25 & 0.20 \\
\hline
\end{tabular}

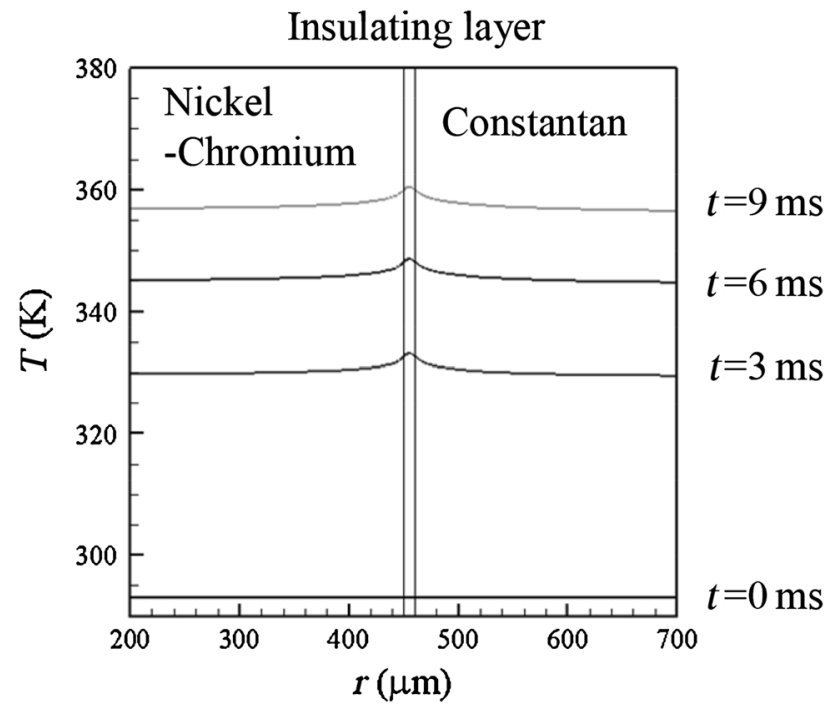

Fig. 4 Temperature change on the surface of the cone heat flux sensor with an input heat flux of $1.0 \mathrm{MW} / \mathrm{m}^{2}$

from with the one-dimensional semi-infinite simplification. If Eq. (4) is still used to calculate the heat flux, the results are as shown in Fig. 5. As Fig. 5 shows, the calculated heat flux is significantly higher than the input value, but basically remains constant. This means that although the insulation layer causes the internal heat conduction of the thermocouple different from one-dimension semi-infinite body, the impact

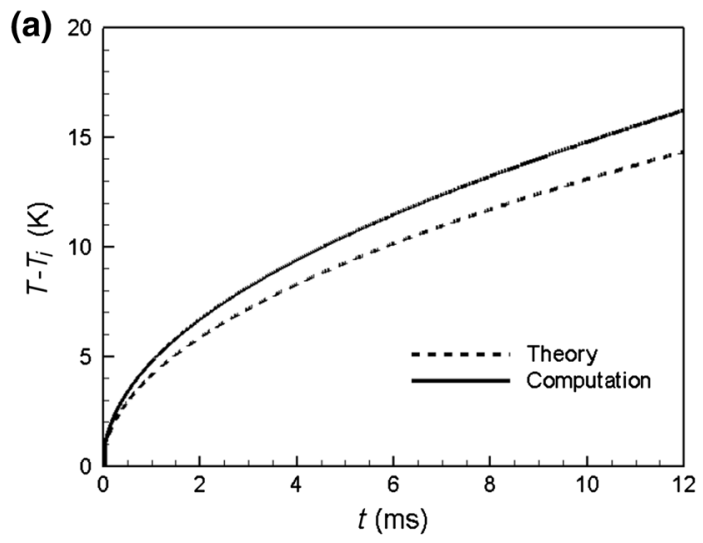

on the calculated heat flux is basically unchanged. If the thickness of the insulating layer can be further reduced, the deviation of the heat flux can be restricted to less than $5 \%$, compared to the assumption of one-dimensional semi-infinite body of homogeneous material.

\section{Measured results of the shock tunnel test}

The Integral cone heat flux sensor has been practically used in JF-8A hypersonic shock tunnel test (Fig. 6) in State Key Laboratory of High Temperature Gas Dynamics, Institute of Mechanics, Chinese Academy of Sciences. In the test, JF-8A can duplicate flow conditions with Mach number $(M)$ from 6.5 to 12 and Reynolds number from $5 \times 10^{5}$ to $3.7 \times 10^{7} / \mathrm{m}$. Its valid test time ranges from 5 to $30 \mathrm{~ms}$.

The main parameters of the flow field in this test are given as follows: the incoming flux $M_{\infty}=6.36$, the total pressure $P_{0}=4.88 \mathrm{MPa}$, the total temperature $T_{0}=560 \mathrm{~K}$, the Reynolds number per unit length $R e_{\infty}=$ $3.2 \times 10^{7} / \mathrm{m}$ and the test time $20 \mathrm{~ms}$ (Fig. 7).

The test data is shown in Table 2, from which it can be seen that the general reproducibility of the integral thermocouple is good, with a relative deviation of less than $10 \%$ for the 7 test results. Over $85 \%$ of the sensors have a deviation of less than $7 \%$, and some of the data has a deviation of less than $1 \%$.

In addition, to predict heat flux at stagnation points, there is the well-known Fay-Riddell formula [10]:

$$
\begin{aligned}
q_{\mathrm{w} 0}= & 0.763 \operatorname{Pr}^{-0.6}\left\{(\rho u)_{\mathrm{w}}^{0.1}(\rho u)_{0}^{0.4}\left[1+\left(L_{\mathrm{e}}^{0.52}-1\right) \frac{h_{\mathrm{D}}}{h_{0}}\right]\right. \\
& \left.\left(h_{0}-h_{\mathrm{w}}\right) \sqrt{\left(\frac{\mathrm{d} u_{\mathrm{e}}}{\mathrm{d} x}\right)_{0}}\right\},
\end{aligned}
$$

where $\rho$ is density, $h$ denotes enthalpy, $u$ is dynamic viscosity coefficient, $P r=0.71$ is Prandtl number, $L_{\mathrm{e}}=1.4$ is

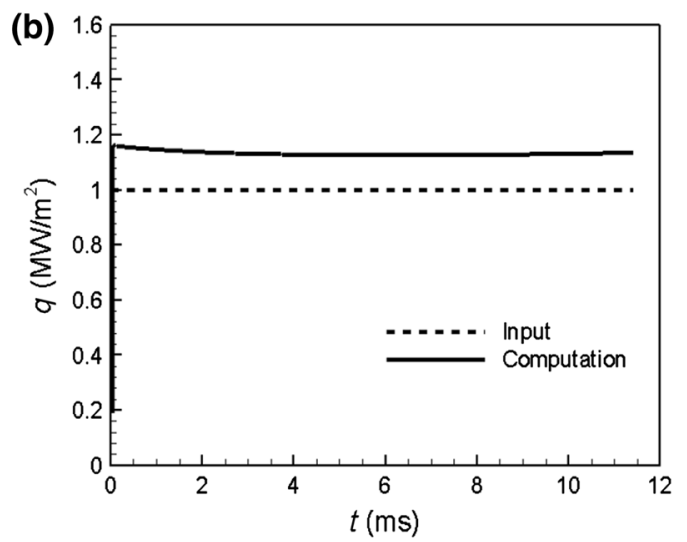

Fig. 5 Time changes of temperature (a) and heat flux (b) 


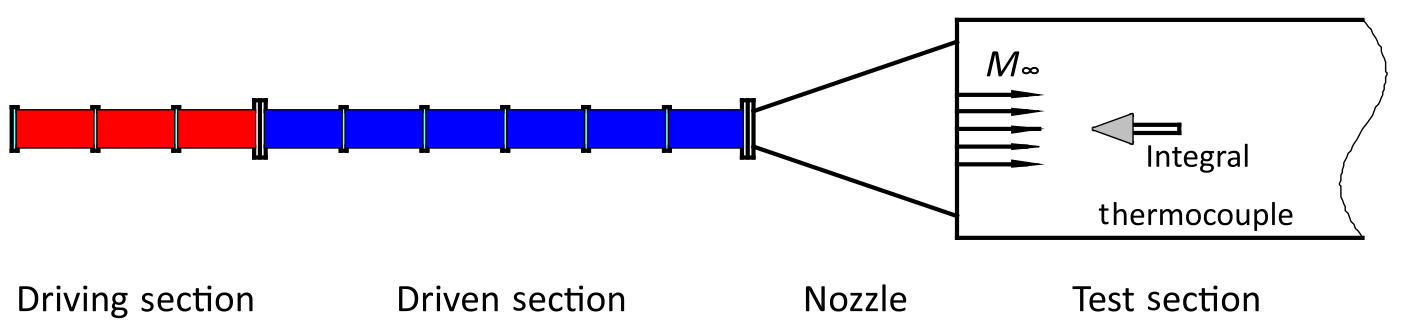

Fig. 6 (Color online) Schematic diagram for the arrangement of the integral thermocouple test in JF-8A
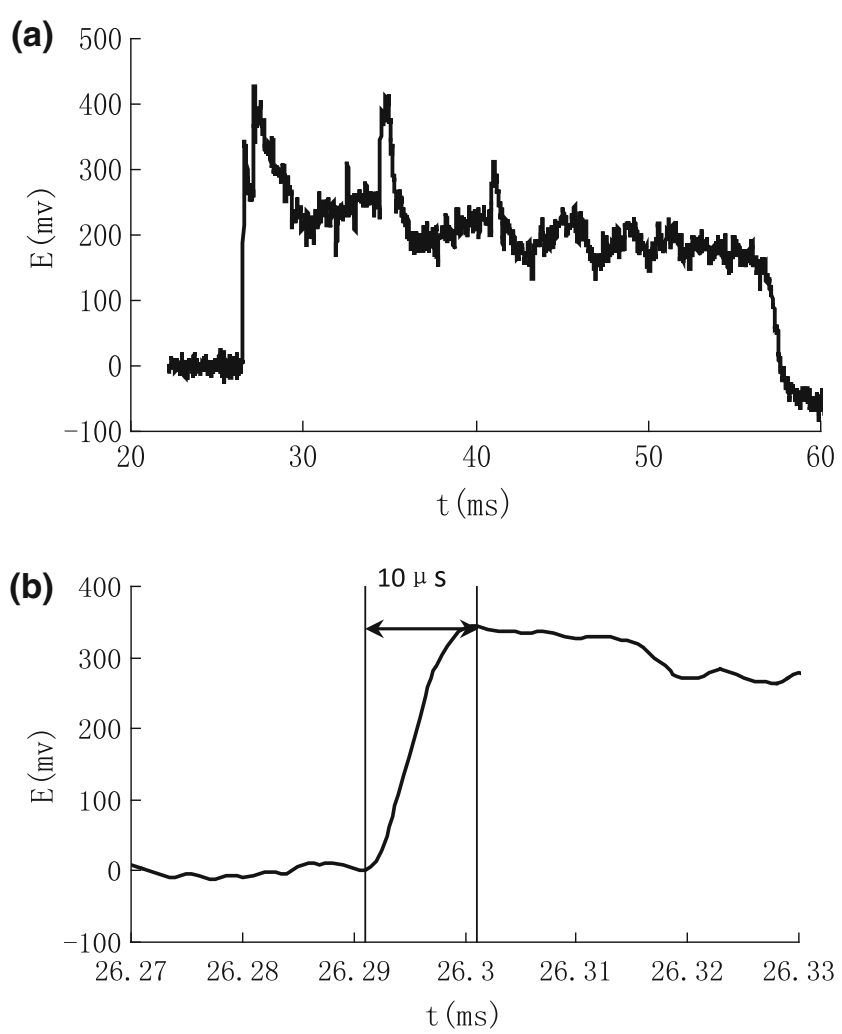

Fig. 7 Heat flux signal curve of the integral thermocouple. a Original electrical signal curve; b Jumping section of electrical signal curve

Lewis number, and $h_{\mathrm{D}}$ the gas dissociation enthalpy. The subscript " $w$ " represents wall, "0" the stagnation point, and " $\infty$ " the incoming condition.

Comparison of the test results with the calculated values obtained from formula (6) is shown in Fig. 8. Under the experimental conditions, the deviation of average value of test data from the calculated value is less than $7 \%$.

The test measurement error consists of sensor, wind tunnel flow field, caused by many factors such as measurement and control system. The main error source of Monolithic thermocouple includes: (a) nickel chromium and constantan are alloy, composition differences cause the error of the dividing value; (b) the influence of the insulation layer junctions, layer temperature; (c) part of the sensor contact layer with poor quality, such as virtual connect.

\section{Discussion}

In this paper, bipropellant electron beam physical vapor deposition is used for preparation of the integral thermocouple heat sensor with constantan thin-film hot junctions. The size of the sensing element can be reduced to approximately $0.1 \mathrm{~mm}$ in minimum, while its spatial resolution has been improved by one magnitude than the traditional thermal sensor, and can be used to measure the heat flux of $R 0.5 \mathrm{~mm}$ cone stagnation point. The frequency response of integral thermocouple heat sensor can reach $100 \mathrm{kHz}$, and is able to completely meet the demands of shock tunnel test with time of only milliseconds.

Integral thermocouple has higher measurement accuracy, and the deviation of the seven test results is less than $10 \%$. Such precision is obviously superior to traditional cylindrical thin platinum film resistance thermometer. There are three main reasons: (1) Metal is used as substrate for the integral thermocouple. With even texture, metal is significantly better than the glass substrate. (2) The integral

Table 2 Measured data $\left(\mathrm{MW} / \mathrm{m}^{2}\right)$ of the heat flux at stagnation points with varied head radiuses

\begin{tabular}{lllllllll}
\hline & No. 1 & No. 2 & No. 3 & No. 4 & No. 5 & No. 6 & No. 7 & Average \\
\hline$q_{0}(R 0.5 \mathrm{~mm})$ & 1.418 & 1.416 & 1.467 & 1.427 & 1.433 & 1.392 & 1.410 & 1.423 \\
$q_{0}(R 1.0 \mathrm{~mm})$ & 1.042 & 1.035 & 0.992 & 1.030 & 0.917 & 0.924 & 0.914 & 0.979 \\
$q_{0}(R 2.0 \mathrm{~mm})$ & 0.632 & 0.612 & 0.616 & 0.623 & 0.688 & 0.701 & 0.677 & 0.650 \\
$q_{0}(R 4.0 \mathrm{~mm})$ & 0.480 & 0.465 & 0.450 & 0.500 & 0.442 & 0.433 & 0.462 & 0.462 \\
$q_{0}(R 5.0 \mathrm{~mm})$ & 0.421 & 0.395 & 0.379 & 0.356 & 0.402 & 0.399 & 0.388 & 0.391 \\
\hline
\end{tabular}




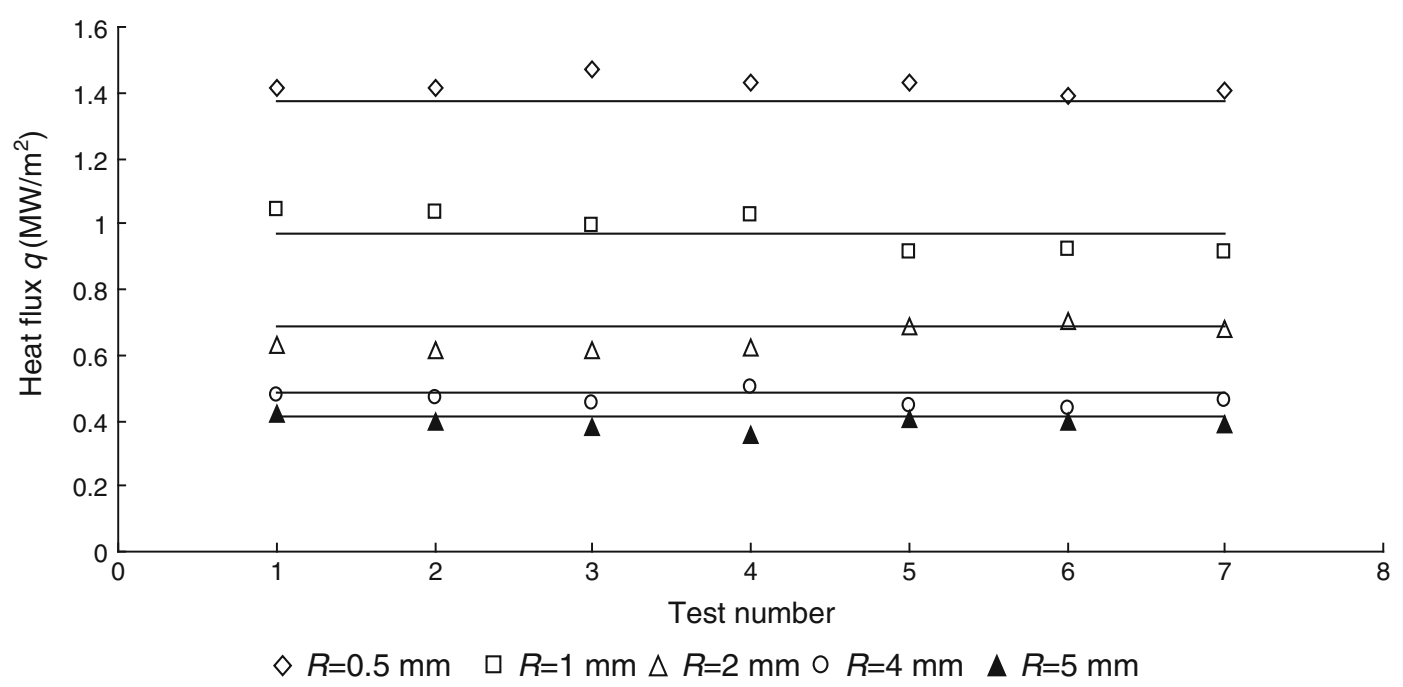

Fig. 8 Comparison of the measured data and theoretical prediction of heat flux at stagnation points with different radiuses (solid line represents calculated value based on Eq. (6))

thermocouple has strong erosion resistance. As long as the poles are connected, the size of the junction is not important. In addition, the adhesion of the metal film to the metal substrate is much better than that of a platinum film coated on a glass substrate. (3) The greatest advantage of the integral thermocouple is a superior guarantee of the model remaining constant in surface shape. Even junction with diameter of $0.1 \mathrm{~mm}$ has a trivial impact on its shape.

However, the sensitivity of the thermocouple is low. When used in the measurement of heat flux of larger diameter ball stagnation point, the signal-to-noise ratio is poor. Therefore, the integral thermocouple is more applicable for measurement of high heat flux, such as that at small-radius ball stagnation point, reattachment zone, and so on. Furthermore, occasionally, the constantan film on very few sensors failed to withstand air scour and peeled off, probably due to surface defects before coating.

Acknowledgments The author wishes to thank Professor Jing Fan for discussion and assistance.

\section{References}

1. Nakakita K, Osafune T, Asai K. Global heat transfer measurement in a hypersonic shock tunnel using temperature-sensitive. AIAA-2003-0743
2. Hubner J, Carroll B, Schanze K et al. Temperature and pressure sensitive paint measurements in short duration hypersonic flow. AIAA-99-0388

3. Oliver H. Thin-film gauges and coaxial thermocouples for measuring transient temperatures. (Description-gauges2.pdf, from http://www.swl.rwth-aachen.de)

4. Holden MS. A database of aerothermal measurements in hypersonic flow in building block experiments for CFD validation. AIAA Paper 2003-1137

5. Zhang SZ, Chen H, Dong ZC et al (2010) Heat flux measurement at stagnation point in high-enthalpy shock tubes. In: Proceedings of the 14th Chinese National Symposium on Shock Waves (in Chinese)

6. Chen X, Gong J, Shi J et al (2012) Experimental study of stagnation point heat transfer measurement on sharp leading edges. In: Proceedings of the 15th Chinese National Symposium on Shock Waves (in Chinese)

7. Schultz DL, Jones TV (1973) Heat-transfer measurements in short-duration hypersonic facilities. AGARD-AG-165

8. Li SH, Shu YH, Fan J (2008) Thickness and component distribution of yttrium-titanium alloy films in electron beam physical vapor deposition. Sci China Ser E Tech Sci 51:1470-1482

9. Fan J, Shu YH, Liu HL (2005) Development of multi-element electron bean physical deposition system. Scientific Report of Institute of Mechanics, Chinese Academy of Science, IMCAS STR 2005001

10. Fay JA, Riddell FR (1958) Theory of stagnation point heat transfer in dissociated air. J Aerosp Sci 25 